\title{
Artículo Original: Frecuencia de malformaciones congénitas detectadas en la Sección de Gineco-obstetricia y Neonatología del Hospital San Juan de Dios durante el periodo comprendido entre enero a diciembre del año 2014
}

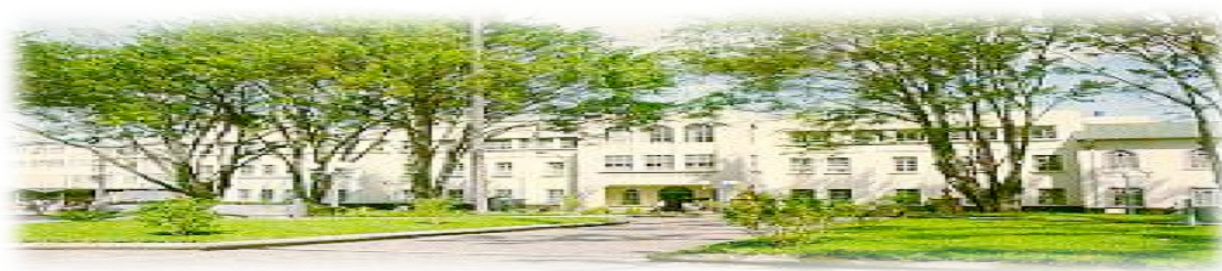

Hospital San quan de Dios, San José, Costa Rica. Fundado en 1845
Recibido: $\quad 14 / 04 / 2016$

Aceptado: $\quad 14 / 04 / 2016$
${ }^{1}$ Amy Carolina Zambrano García

${ }^{2}$ Joaquín Bustillos Villavicencio ${ }^{3}$ Pablo Parra Ramírez

${ }^{4}$ Verónica Saborío López ${ }^{5}$ Eugenio Calderón Solano ${ }^{6}$ Jorge Arturo Mora Sandí

\footnotetext{
${ }^{1}$ Médico Residente Ginecología-Obstetricia. Hospital Materno Infantil José Domingo De Obaldía, David, Panamá, CSS

${ }^{2}$ Médico Asistente Especialista Medicina Materno Fetal, Hospital San Juan de Dios, San José, Costa Rica, CCSS
}

RESUMEN

Al revisar los casos registrados de malformaciones fetales durante el año 2014, nos brinda una perspectiva de cuáles tipos de dismorfismos estamos detectando en nuestro medio y su frecuencia. Se trata de un estudio descriptivo de forma retrospectivo en el Hospital San Juan de Dios, San José, Costa Rica, con un total de 54 nacidos vivos durante el período de Enero a Diciembre del año 2014, a los cuales se les diagnosticó al nacimiento determinada malformación congénita, simple o múltiple y que hubiesen nacido ya sea por parto o cesárea. El criterio de inclusión son los productos que nacieron con una o más malformaciones congénitas durante el período Enero a Diciembre de 2014, en el Hospital San Juan de Dios. Se excluyeron pacientes que no presentaban datos completos en la boleta de notificación del Hospital San Juan de Dios.

Se reportaron en dicho periodo 5004 nacimientos, de los cuales 4960 fueron nacidos vivos, y de éstos 54 presentaron malformaciones congénitas. Las malformaciones congénitas fueron clasificadas por sistema, se obtuvo como resultado que las de mayor incidencia fueron las del sistema nervioso 
central, 14 casos para un $22.9 \%$, le siguen las del sistema genitourinario y músculo esqueléticas con 10 casos, $16.3 \%$ respectivamente. Del sistema gastrointestinal se presentaron 9 casos para un $14.7 \%$, dentro de los síndromes se presentaron 9 casos, siendo el de mayor incidencia síndrome de Down (trisomía 21), 6 casos, además síndrome de Edward, Patau y Peters Plus, las de menor incidencia fueron las cardiovasculares, 5 casos $(8.1 \%)$, craneofaciales 3 casos $(4.9 \%)$ y del sistema respiratorio 1 caso. En nuestro estudio, la incidencia de malformaciones congénitas en el año 2014 fue $1.7 \%$, siendo las más frecuentes las del sistema nervioso central, luego las músculo esqueléticas y genitourinarias. Las del sistema cardiovascular representaron una baja incidencia $8.1 \%$ del total y el área de mayor procedencia de madres con productos con malformaciones congénitas fue Santa Ana en la provincia de San José.

\section{PALABRAS CLAVE}

Feto. Malformaciones; cardiopatías. Sistema nervioso central. Genitourinarias

\section{ABSTRACT}

Review of the recorded cases of fetal malformations during 2014 gives us a perspective of what types of dysmorphisms we are detecting in our environment and their frequency. This is a descriptive study on a retrospective at the San Juan de Dios Hospital, San José, Costa Rica, with a total of 54 live births during the period from January to December 2014, to which were diagnosed at certain birth defect congenital, single or multiple, and were born either by vaginal delivery or caesarean section. The criteria for inclusion are products that were born with one or more birth defects during the period January to December 2014, in the San Juan de Dios Hospital. Patients who did not have complete data on the notification card of the Hospital San Juan de Dios were excluded.
They were reported in the period 5004 births, of which 4960 were born alive, and of these 54 had congenital malformations. Congenital malformations were classified by system, it resulted that the central nervous system were the ones with highest incidence, 14 cases representing the $22.9 \%$, followed by the genitourinary system and skeletal muscle with 10 cases, representing the $16.3 \%$ respectively. 9 cases of gastrointestinal system, representing the $14.7 \%$, 9 cases were presented within the syndromes, being Down syndrome the one with highest incidence (trisomy 21), 6 cases also presented Edward syndrome and Patau Peters Plus, the ones with lowest incidence of all were cardiovascular malformations, with 5 cases, $(8.1 \%)$, craniofacial in 3 patients $(4.9 \%)$, and 1 case respiratory system. In our study, the incidence of congenital malformations in 2014 was $1.7 \%$, being central nervous system malformations the most frequent, followed by musculoskeletal and genitourinary. The cardiovascular system represented a low incidence pf $8.1 \%$ and the largest area of origin of mothers with products with congenital malformations was Santa Ana, located in the province of San José.

\section{KEY WORDS}

Fetus. Malformations. Heart disease. Central nervous system. Genitourinary

\section{INTRODUCCIÓN}

El presente trabajo consiste en brindar una perspectiva general de la frecuencia de las principales malformaciones congénitas en el Hospital San Juan de Dios (HSJD), San José Costa Rica, durante el período de Enero a Diciembre del año 2014.

Las anomalías congénitas, también llamadas defectos de nacimiento, trastornos congénitos o malformaciones congénitas, pueden ser estructurales, pero también funcionales, como ocurre con los trastornos metabólicos presentes desde el nacimiento. 
La Organización Mundial de la Salud (OMS) define las malformaciones congénitas como toda aquella anomalía del desarrollo morfológico, estructural, funcional o molecular que esté en un niño recién nacido, sea externa o interna, familiar o esporádica, hereditaria o no, única o múltiple, que resulta de una embriogénesis defectuosa. La guía de referencia de uso más común para clasificar los defectos de nacimiento es la Clasificación Internacional de Enfermedades, (CIE-10 Q000- Q999). ${ }^{1,2}$

No es posible asignar una causa específica a cerca de un $50 \%$ de las anomalías congénitas. No obstante, se han identificado algunas de sus causas o factores de riesgo. Los recientes avances en el campo de la genética han permitido caracterizar y darle la importancia a las alteraciones de los genes en los procesos de desarrollo de las enfermedades del ser humano, especialmente de las alteraciones que desencadenan malformaciones. Pero hoy, se conocen otros determinantes de salud que también influyen, como los estilos de vida, el medio ambiente, la atención en salud, la nutrición (especialmente el ácido fólico y el yodo), el riesgo derivado de la edad, la inmunidad y las enfermedades crónicas más prevalentes en la mujer gestante y su repercusión sobre el feto.

El factor socioeconómico puede tratarse de un determinante indirecto, las anomalías congénitas son más frecuentes en las familias y países con escasos recursos. Se calcula que aproximadamente un $94 \%$ de los defectos de nacimiento graves se producen en países de ingresos bajos y medios, en los que las madres son más vulnerables a la malnutrición, tanto por macronutrientes como por micronutrientes, y pueden tener mayor exposición a agentes o factores que inducen o aumentan la incidencia de un desarrollo prenatal anormal, en especial el alcohol y las infecciones. La edad materna avanzada también incrementa el riesgo de algunas alteraciones cromosómicas, como el síndrome de Down.

La consanguineidad aumenta la prevalencia de anomalías congénitas genéticas raras y multiplica por cerca de dos el riesgo de muerte neonatal e infantil, discapacidad intelectual y anomalías congénitas graves en los matrimonios entre primos hermanos. Algunas comunidades étnicas, como los judíos asquenazíes o los finlandeses, tienen una mayor prevalencia de mutaciones genéticas raras que condicionan un mayor riesgo de anomalías congénitas.

Las infecciones maternas, como la sífilis o la rubéola, son una causa importante de defectos de nacimiento en los países de ingresos bajos $\mathrm{y}$ medios.

En cuanto al estado nutricional de la madre las carencias de yodo y folato, el sobrepeso y enfermedades como la diabetes mellitus están relacionadas con algunas anomalías congénitas. Por ejemplo, la carencia de folato aumenta el riesgo de tener niños con defectos del tubo neural. Es importante evitar la exposición materna a pesticidas, fármacos y drogas, alcohol, tabaco, productos químicos, altas dosis de vitamina A al inicio del embarazo y altas dosis de radiación, ya que aumentan el riesgo de que los niños nazcan con anomalías congénitas. El hecho de trabajar en basureros, fundiciones $o$ minas o de vivir cerca de esos lugares también puede ser un factor de riesgo.

Las anomalías congénitas se pueden clasificar desde dos grandes puntos de vista. El primero se refiere a la severidad de la anomalía, el segundo se refiere a la naturaleza y origen de la anomalía. ${ }^{3}$

Cuando no generan mortalidad, estos defectos pueden desencadenar una serie de eventos crónicos en la persona que las padece, los cuales no solo afectan su calidad de vida, sino también la de sus cuidadores.

En cifras aproximadas, las anomalías congénitas (también llamadas defectos de nacimiento) afectan a uno de cada 33 lactantes y causan 3,2 millones de discapacidades al año. Se calcula que cada año 270000 recién nacidos fallecen durante los primeros 28 días de vida debido a anomalías congénitas. Las anomalías congénitas pueden ocasionar discapacidades crónicas con gran impacto en los afectados, sus familias, los sistemas de salud y la sociedad. Los trastornos congénitos graves más frecuentes son las malformaciones cardíacas, los defectos del tubo neural y el síndrome de Down. ${ }^{4,5}$

Al igual que en Costa Rica, en varios países industrializados la disminución de la mortalidad infantil por enfermedades infecciosas ha provo- 
cado que otros padecimientos como las malformaciones congénitas aparezcan entre las primeras causa de mortalidad. En Estados Unidos, los defectos congénitos ocupan actualmente el primer lugar en la etiología de las muertes infantiles 6,7, lo mismo en Barcelona (España), donde también encabezan las causas de defunciones infantiles, seguidas por las enfermedades perinatales ${ }^{8}$. Por otro lado, en países latinoamericanos donde la mortalidad infantil ha caído por debajo de 50 por 1000 nacimientos, las anomalías congénitas ocupan el tercer puesto en la mortalidad infantil y han adquirido significancia como problema de salud pública. ${ }^{8,9}$

La atención preconceptiva y periconceptiva consta de prácticas básicas de salud reproductiva, así como de un examen médico y pruebas genéticas que se pueden realizar a lo largo del embarazo. En el periodo preconceptivo, para identificar a las personas en riesgo de padecer determinados trastornos o de transmitirlos a sus hijos. La estrategia consiste en el uso de los antecedentes familiares y la detección del estado de portador, y es particularmente valiosa en países en los que el matrimonio consanguíneo es frecuente. En el periodo antenatal, para detectar la edad materna avanzada, la incompatibilidad $\mathrm{Rh}$ y los estados de portador. La ecografía permite detectar el síndrome de Down durante el primer trimestre y las anomalías fetales graves durante el segundo trimestre; los análisis del suero materno también permiten detectar el síndrome de Down y los defectos del tubo neural durante el primero y el segundo trimestres. El advenimiento de la ecografía en la medicina fetal constituye actualmente una de las herramientas más importantes para el diagnóstico prenatal. El examen ecográfico detallado de la anatomía fetal permite detectar, por un lado, las malformaciones y por otro, los marcadores ecográficos. En el periodo neonatal, para detectar trastornos hematológicos, metabólicos y hormonales. Las pruebas para detectar la sordera y las malformaciones cardíacas, y la detección precoz de los defectos de nacimiento pueden facilitar la instauración de tratamientos capaces de salvar la vida y prevenir la progresión hacia discapacidades físicas, intelectuales, visuales o auditivas.

A pesar de que hoy en día se dispone de diferentes técnicas y exámenes que pueden ayudar a identificar una anomalía congénita desde la gestación, la gran mayoría de éstas, se diagnostican en el momento del nacimiento a través del examen físico, y pruebas paraclínicas. Algunas permanecen ocultas por muchos años durante la vida del afectado. ${ }^{6}$

Durante la gestación, la principal herramienta diagnóstica de estas alteraciones sigue siendo la ecografía, sin embargo, su rendimiento diagnóstico es muy bajo, debido a las características operacionales de esta técnica en una mujer embarazada, además de depender también de la habilidad y experticia del médico que lo practica. Otros exámenes como amniocentesis, biopsia de vellosidades coriónicas, entre otros, son más costosos y de poca a nula utilidad cuando no se tiene ninguna sospecha de alguna anomalía.

En cuanto al tratamiento, existe un rango de opciones que va de acuerdo al tipo de anomalía según su severidad y naturaleza. Algunas de las más leves, no requieren más allá de un seguimiento en los primeros meses de vida, con la alta probabilidad de no generar ninguna secuela permanente en la vida del paciente y sus familiares. Otras por el contrario, van a requerir manejos más agresivos desde el momento del nacimiento, o incluso desde antes de nacer, como procedimientos quirúrgicos, administración de fármacos y monitoreo estricto por médicos especializados en unidades diseñadas para este tipo de pacientes. $^{6}$

\section{MATERIALES Y MÉTODOS}

Se realizó un estudio descriptivo, retrospectivo en el Hospital San Juan de Dios, San José, Costa Rica, basado en un total de 54 nacidos vivos durante el período de Enero a Diciembre del año 2014, a los cuales se les diagnosticó al nacimiento determinada malformación congénita, simple o múltiple y que hubiesen nacido ya sea por parto vaginal o cesárea.

Los datos fueron tomados de la boleta de notificación obligatoria de malformaciones congénitas del Hospital San Juan de Dios.

Se analizaron variables maternas y fetales. Entre las maternas: edad, procedencia, aplicación de vacuna anti-rubéola y edad gestacional; fetales: 
sexo, peso, malformación por sistema, y su destino al momento del diagnóstico.

\section{OBJETIVO}

Conocer la frecuencia de las malformaciones congénitas de los neonatos nacidos en el Hospital San Juan de Dios, San José, Costa Rica, durante el período de Enero a Diciembre de 2014.

\section{CRITERIOS DE INCLUSIÓN}

Todo producto que nació con una o más malformaciones congénitas durante el período Enero a Diciembre de 2014, en el Hospital San Juan de Dios.

\section{CRITERIOS DE EXCLUSIÓN}

Se excluyeron pacientes que no presentaban datos completos en la boleta de notificación del Hospital San Juan de Dios.

\section{RESULTADOS}

Durante el período de estudio, Enero a Diciembre de 2014, se reportaron 5004 nacimientos, de los cuales 4960 fueron nacidos vivos, y de éstos 54 presentaron malformaciones congénitas (Tabla I).

El grupo de edad materna con mayor frecuencia de alguna malformación fue el comprendido entre 21-25 años, correspondiendo a 14 recién nacidos afectados, $26.9 \%$. Se excluyeron dos pacientes a las cuales no se les consignaba la edad en la boleta de notificación (Tabla II).

La mayoría de las madres eran procedentes de la provincia de San José, 49 para un 92.4\% del total, con una mayor incidencia en el cantón Central 14 para un $26.4 \%$, seguido por Santa Ana 10 casos para un $18.8 \%$, luego Alajuelita y Escazú con igual incidencia 7 casos para un 13.2\%, Mora con 5 casos para un $9.4 \%$, Puriscal y Pérez Zeledón con 2 casos para un $3.7 \%$, Curridabat y Desamparados con un caso para cada cantón. El resto de los casos se presentaron uno en cada región de las siguientes: Golfito, Osa, Buenos Aires, Unión y Limón (Tabla III).

De los casos reportados 8 eran de nacionalidad nicaragüense y procedentes en su mayoría de Santa Ana.

En cuanto a la información correspondiente a si la madre recibió la vacuna anti rubéola en el embarazo, 3 casos $(5.5 \%)$ refirieron haber recibido la vacuna, el resto no se consignaba la información (Tabla I).

De acuerdo a la edad gestacional la mayor incidencia de los casos se presentó en las pacientes con embarazo entre 37 y 41 semanas, 35 casos para un $64.8 \%$, luego 14 pacientes $25.9 \%$ para embarazos entre 34.1 y 36.6 semanas y 5 casos $9.2 \%$ para menores de 34 semanas (Tabla IV).

Las malformaciones congénitas fueron clasificadas por sistema, se obtuvo como resultado que las de mayor incidencia fueron las del sistema nervioso central, 14 casos para un $22.9 \%$, le siguen las del sistema genitourinario y músculo esqueléticas con 10 casos, 16.3\% respectivamente. Del sistema gastrointestinal se presentaron 9 casos para un $14.7 \%$, dentro de los síndromes se presentaron 9 casos, siendo el de mayor incidencia síndrome de Down (Trisomía 21) 6 casos, además síndrome de Edward, Patau y Peters Plus, las de menor incidencia fueron las cardiovasculares, 5 casos, $8.1 \%$, craneofaciales 3 casos, $4.9 \%$ y del sistema respiratorio 1 caso (Tabla V).

En relación al sexo del recién nacido, de los 54 casos reportados, $33(61.1 \%)$ fueron del sexo masculino, 19 (35.2\%) femeninos y 2 casos ambiguos. Todos fueron recién nacidos vivos (Tabla VI).

De acuerdo al peso del recién nacido, la mayor frecuencia de malformaciones estuvo en el rango de $>2000$ g, 44 casos, 81.4\% (Gráfico 1).

\section{DISCUSIÓN}

Al ser este un trabajo sobre incidencia de malformaciones congénitas, en un período de un año, trataremos de cotejar nuestros resultados 
con estudios similares. En cuanto a incidencia, la literatura describe que la frecuencia de defectos congénitos en Estados Unidos y otros países del mundo representan menos del 5\% de los nacimientos, lo que coincide con nuestro estudio, en el que el resultado fue $1.7 \%$. $10,11,12,13,14,15$

La edad materna se refiere por la mayoría de los autores, que se presenta entre los 21 y 25 años; lo que coincide con este estudio, 26.9\%, seguido por el grupo de 26-30 años, que representa el $25 \%$. ${ }^{16}$

En relación a la procedencia de la madre, la mayoría de los casos se encontraron procedente de la provincia de San José, específicamente del área de Santa Ana, representando el 92.4\% de los casos. El $15.1 \%$ de los casos fueron de nacionalidad nicaragüense.

La diferencia de sexo entre los pacientes con algún tipo de malformación congénita fue mayor la frecuencia en los pacientes de sexo masculino, $61.1 \%$. Este resultado coincide con lo reportado en la literatura mundial, en la que se encuentra que las malformaciones congénitas afectan en mayor proporción a los hombres que a las mujeres, con una relación hombre/mujer de $1,06^{4}$ y en nuestro estudio 1.7.

La mayoría de los casos, $27.7 \%$ tuvieron un peso que fluctuaba de 2,501 a 3,000 gramos, este resultado difiere a estudios similares que reportan un riesgo 6 veces mayor de malformaciones en aquellos con un peso menor a $\operatorname{los} 1500 \mathrm{~g}^{14}$, de acuerdo a la edad gestacional, la literatura indica una mayor incidencia en los pre término a diferencia de nuestro estudio en el cual la frecuencia mayor fue en edad gestacional de 37-41 semanas.

De manera global, en orden de frecuencia, el primer lugar lo comparten, con un $21 \%$ del total, las malformaciones cardíacas y genitourinarias, siguiendo en frecuencia con un 16\% las del sistema nervioso central y luego las musculo esqueléticas, faciales y gastrointestinales con un 5 a $7 \%$ cada una ${ }^{12,13,14}$.

Los resultados de nuestro estudio muestran que la mayor frecuencia de malformaciones fueron las del Sistema nervioso Central que corresponde a un $22.9 \%$ de éstas la más frecuente labio leporino. Continúan en frecuencia las del sistema genitourinario y músculo esquelético cada una de las cuales representa un $16.3 \%$. Las malformaciones gastrointestinales, a diferencia de otros estudios representaron una mayor frecuencia, la cual fue $14.7 \%$ y las cardíacas que representan las de mayor frecuencia, sólo resultó $8.1 \%$, seguidas por las craneoencefálicas, y del sistema respiratorio.

Las relacionadas a síndromes fueron $14.7 \%$ y de éstos el más frecuente el síndrome de Down, $9.8 \%$, resultado que difiere con el estudio de ECLAM realizado en Chile en el año $2010^{3}$, donde este trastorno genético fue el más frecuente de todas las malformaciones. El destino del recién nacido fue: $32.6 \%$ se les dio egreso a casa, $39.1 \%$ fueron trasladados al Hospital Nacional de Niños, para su tratamiento y $28.3 \%$ fallecieron, los cuales todos presentaban múltiples malformaciones.

\section{CONCLUSIONES}

En nuestro estudio la incidencia de malformaciones congénitas en el año 2014 fue $1.7 \%$, siendo las más frecuentes las del sistema nervioso central, luego las músculo esqueléticas y genitourinarias. Las malformaciones del sistema cardiovascular representaron una baja incidencia $8.1 \%$ y el área de mayor procedencia de madres con productos con malformaciones congénitas fue Santa Ana en la provincia de San José.

\section{RECOMENDACIONES}

Las medidas de salud pública preventivas adoptadas en los periodos preconceptivo $\mathrm{y}$ periconceptivo y los servicios de atención prenatal reducen la frecuencia de algunas anomalías congénitas. Consideramos que se debería:

- Agregar en la boleta de notificación de malformaciones congénitas, si la madre recibió o no ácido fólico, debido a la importancia que éste tiene al prevenir malformaciones del sistema nervioso central, específicamente del tubo neural. 
- Mejorar la dieta de las mujeres en edad fecunda, garantizando una ingesta dietética suficiente de vitaminas y minerales, tales como el ácido fólico y el yodo, y la restricción del consumo de sustancias nocivas, en particular el abuso de alcohol, así como el control de la diabetes antes de la concepción y durante la gestación mediante el asesoramiento, el control del peso, la dieta y la administración de insulina cuando sea necesaria.

- Resulta de gran importancia la mejora de la cobertura con vacunas, en especial contra el virus de la rubéola, en las niñas y las mujeres, se debe hacer énfasis en la consignación de dicho dato en la boleta de notificación. La rubéola es prevenible mediante vacunación. La vacuna anti rubéola puede administrarse al menos 1 mes antes del embarazo en mujeres que todavía no sean inmunes.

- El aumento y el fortalecimiento de la formación del personal de salud y de otros interesados en el fomento de la prevención de los defectos de nacimiento.

- Brindar a los padres asesoramiento genético en el cual estos sean orientados ante la llegada del siguiente embarazo.

\section{BIBLIOGRAFÍA}

1. OMS Anomalías congénitas Nota descriptiva $\mathrm{N}^{\circ} 370$. Abril de 2015

2. March of Dimes Birth Defects Foundation. Global report on birth defects. New York: White Plañís; 2006. Fecha de consulta: abril de 2009. Disponible en:

www.marchofdimes.com/professionals/ 871 18587.asp

3. Clasificación Internacional de Enfermedades, CIE 10. [citado 26 feb 2014]

4. Nazer J Cifuentes L . Malformaciones congénitas en Chile. Período 2001-2010
5. Moore KL. Persaud MD. Embriología Clínica. 6ta. Ed. Interamericana; 175210

6. Medwave 2012 Oct;12(9):e5537 doi: 10.5867/medwave.2012.09.5537

7. Albacar E Borrell C. Evolución de la mortalidad infantil en la ciudad de Barcelona. Gac Sanit. 2004;18(1):24-31

8. García H Salguero GA Moreno J, Arteaga C Giraldo A. Frecuencia de anomalias congénitas en el Instituto Materno Infantil de Bogotá. Biomedica. 2003;23:161-172

9. Barboza-Arguello MP, Benavides-Lara A, Umaña L, Vargas-Leitón B. Mortalidad infantil por defectos congénitos en Costa Rica, 1981-2010. Rev Panam Salud Pública. 2013;34(5):304-311

10. Castilla E Orioli IM. ECLAMC: The Latin-American collaborative study of congenital malformations. Community Genet.;7:76-94

11. Sekhobo J Druschel C. An evaluation of congenital malformations surveillance in New York State: An application for Centers for Disease Control and Prevention (CDC) guidelines for evaluating surveillance systems. Public Health Rep. ;116:296-305

12. Nazer J Aravena T Cifuentes L. Malformaciones congénitas en Chile. Un problema emergente (periodo 19951999). Rev Méd Chile. 2001;129:895904

13. International Clearinghouse for Birth Defects Monitoring Systems (ICBDMS). Annual Report 2006. Fecha de consulta: marzo de 2009. Disponible en: http://www.icbdsr.org/page.asp?p=9895 $\underline{\& l=1}$

14. Gómez J Fernández N Zarante I. Detección de anomalías congénitas en 
15. 12.760 nacimientos de tres hospitales de la ciudad de Bogotá 2004-2005 mediante ecografía prenatal. Rev Colomb Obstet Ginecol. 2007;58:194-201

16. Genética y biomedicina molecular: http://www.uanl.mx/publicaciones/espe ciales/geneticaclínica.html

17. Pachajoa H AY Isaza C. et al. Defectos congénitos mayores en un hospital de tercer nivel en Cali, Colombia 20042008. Rev salud pública. 2010;13(1):152-162

\section{AGRADECIMIENTOS}

Agradecemos enormemente al Servicio de Neoanatología del HSJD y especialmente al soporte secretarial recibido para la obtención de los datos analizados.

\section{CONFLICTO DE INTERÉS Y/O AGRADE- CIMIENTOS}

Los autores declaran que no existió ningún conflicto de interés en el presente reporte.

\section{APÉNDICE}

Tabla I. Generalidades, según Neonatología HSJD.

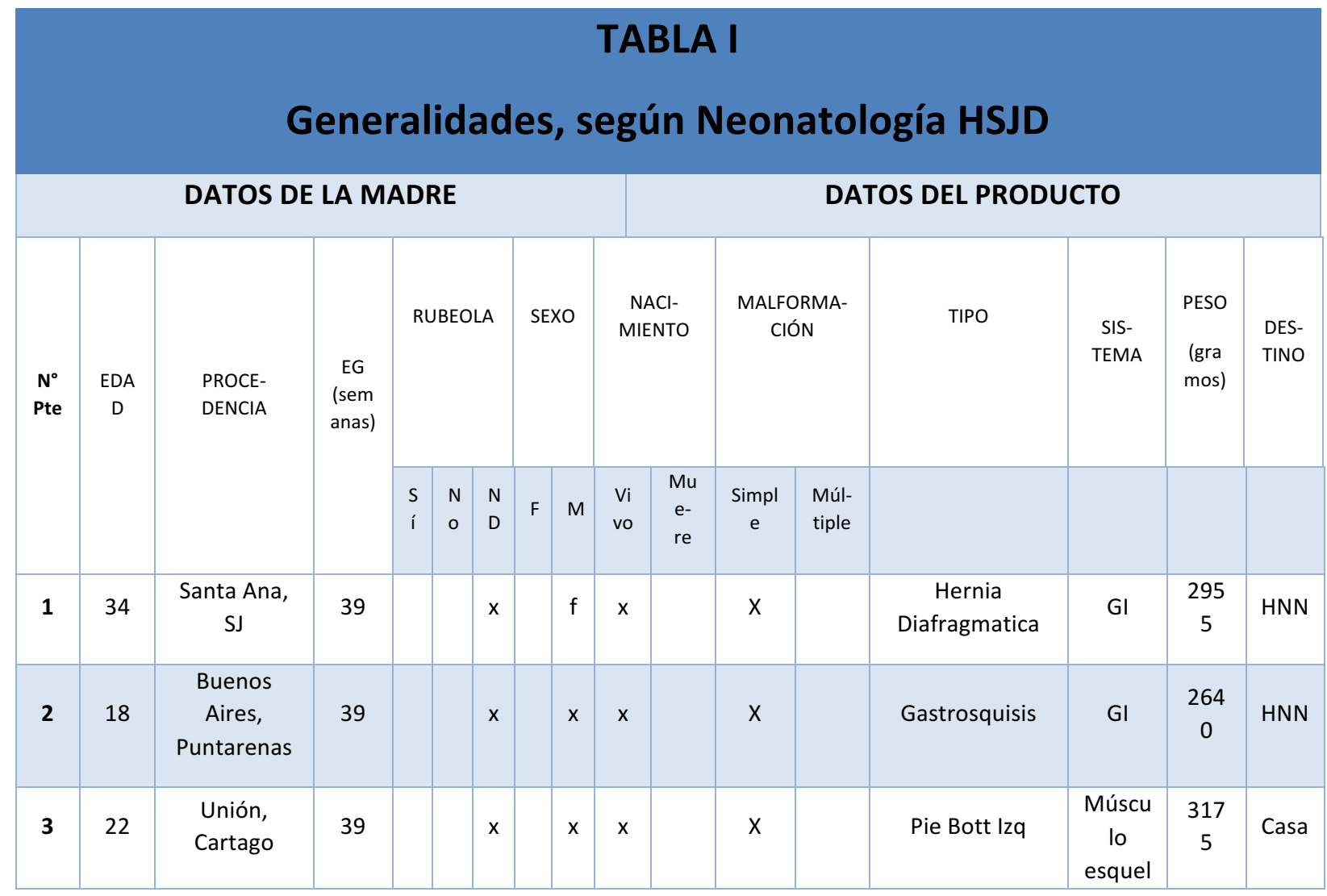




\begin{tabular}{|c|c|c|c|c|c|c|c|c|c|c|c|c|c|c|}
\hline & & & & & & & & & & & & ético & & \\
\hline 4 & 25 & Alajuelita, SJ & 39 & & $x$ & & $\mathrm{x}$ & $\mathrm{x}$ & $x$ & & $\begin{array}{c}\text { Acortamiento } \\
\text { brazo D y mano } \\
\text { hipotrofica }\end{array}$ & $\begin{array}{l}\text { Múscu } \\
\text { lo } \\
\text { esquel } \\
\text { ético }\end{array}$ & $\begin{array}{c}276 \\
0\end{array}$ & Casa \\
\hline 5 & 14 & Escazú,SJ & 34 & & $\mathrm{x}$ & $\mathrm{x}$ & & $\mathrm{x}$ & & $\mathrm{x}$ & $\begin{array}{c}\text { Anomalía } \\
\text { Ebstein, } \\
\text { Hipoplasia pulm } \\
\text { bil,Microcef }\end{array}$ & $\begin{array}{l}\text { CVC } \\
\text { Resp }\end{array}$ & $\begin{array}{c}223 \\
0\end{array}$ & $\begin{array}{l}\text { Falle } \\
\text { ce }\end{array}$ \\
\hline 6 & 24 & $\begin{array}{l}\text { Santa Ana, } \\
\text { SJ }\end{array}$ & 32 & & $\mathrm{x}$ & $\mathrm{x}$ & & $\mathrm{x}$ & & $\mathrm{x}$ & $\begin{array}{c}\text { Microcef,Ventri } \\
\text { cbil, } \\
\text { Megacisterna } \\
\text { Magna }\end{array}$ & SNC & $\begin{array}{c}164 \\
0\end{array}$ & HNN \\
\hline 7 & 28 & Central,SJ & 40 & & $\mathrm{x}$ & $\mathrm{x}$ & & $\mathrm{x}$ & & $\mathrm{x}$ & $\begin{array}{l}\text { Agenesia renal } \\
\text { nil,Dismorfismo: } \\
\text { orejas implant } \\
\text { baja,frontal } \\
\text { amplio,aplanam } \\
\text { iento nasal, } \\
\text { mamilas } \\
\text { separadas }\end{array}$ & $\begin{array}{l}\text { Apto } \\
\text { Genit } \\
\text { o- } \\
\text { urinari } \\
\text { o } \\
\text { Sínd. } \\
\text { Down }\end{array}$ & $\begin{array}{c}193 \\
0\end{array}$ & $\begin{array}{l}\text { Falle } \\
\text { ce }\end{array}$ \\
\hline 8 & 30 & $\begin{array}{c}\text { Osa, } \\
\text { Puntarenas }\end{array}$ & 38 & & $\mathrm{x}$ & $\mathrm{x}$ & & $\mathrm{x}$ & & $\mathrm{x}$ & $\begin{array}{c}\text { Onfalocele } \\
\text { gigante, } \\
\text { agenesia cadera } \\
\text { izq y muslo, Pie } \\
\text { Bott bil, Espina } \\
\text { bífida }\end{array}$ & $\begin{array}{l}\text { Múscu } \\
\text { lo } \\
\text { esquel } \\
\text { ético } \\
\text { SNC }\end{array}$ & $\begin{array}{c}361 \\
5\end{array}$ & $\begin{array}{l}\text { Falle } \\
\text { ce }\end{array}$ \\
\hline 9 & 22 & $\begin{array}{l}\text { Santa Ana, } \\
\text { SJ }\end{array}$ & 37 & $\mathrm{x}$ & & & $\mathrm{x}$ & $\mathrm{x}$ & $\mathrm{x}$ & & $\begin{array}{c}\text { Uretero } \\
\text { hidronefrosis D }\end{array}$ & GU & $\begin{array}{c}331 \\
5\end{array}$ & Casa \\
\hline 10 & 18 & Alajuelita, SJ & 37 & $\mathrm{x}$ & & $\mathrm{x}$ & & $\mathrm{x}$ & & $\mathrm{x}$ & $\mathrm{T}-13$ & $\begin{array}{l}\text { Sínd. } \\
\text { Patau }\end{array}$ & $\begin{array}{c}209 \\
0\end{array}$ & $\begin{array}{l}\text { Falle } \\
\text { ce }\end{array}$ \\
\hline 11 & 20 & Puriscal, SJ & 38 & $\mathrm{x}$ & & & $\mathrm{x}$ & $\mathrm{x}$ & & $\mathrm{x}$ & $\begin{array}{c}2 \text { vasos } \\
\text { umbilicales, } \\
\text { Apéndice pre } \\
\text { auricular gde }\end{array}$ & $\begin{array}{l}\text { CVC } \\
\text { Crane } \\
\text { o } \\
\text { facial }\end{array}$ & $\begin{array}{c}329 \\
0\end{array}$ & $\begin{array}{l}\text { Falle } \\
\text { ce }\end{array}$ \\
\hline 12 & 23 & Central, SJ & 38 & & $\mathrm{x}$ & & $\mathrm{x}$ & $\mathrm{x}$ & & $\mathrm{x}$ & $\begin{array}{l}\text { Labio y paladar } \\
\text { hendidos }\end{array}$ & SNC & $\begin{array}{c}286 \\
0\end{array}$ & Casa \\
\hline 13 & 33 & Escazú,SJ & 36 & & $x$ & $\mathrm{x}$ & & $\mathrm{x}$ & $\mathrm{x}$ & & $\begin{array}{l}\text { Apéndice } \\
\text { preauric bil }\end{array}$ & $\begin{array}{l}\text { Crane } \\
\text { o } \\
\text { facial }\end{array}$ & $\begin{array}{c}216 \\
5\end{array}$ & Casa \\
\hline 14 & 28 & Central, SJ & 40 & & $\mathrm{x}$ & $\mathrm{x}$ & & $\mathrm{x}$ & $\mathrm{x}$ & & $\begin{array}{l}\text { Polidactilia } \\
\text { mano D }\end{array}$ & $\begin{array}{l}\text { Múscu } \\
\text { lo } \\
\text { esquel }\end{array}$ & $\begin{array}{c}342 \\
0\end{array}$ & Casa \\
\hline
\end{tabular}




\begin{tabular}{|c|c|c|c|c|c|c|c|c|c|c|c|c|c|}
\hline & & & & & & & & & & & ético & & \\
\hline 15 & 32 & $\begin{array}{l}\text { Santa Ana, } \\
\text { SJ }\end{array}$ & 34 & $x$ & $x$ & & $x$ & $x$ & & $\begin{array}{c}\text { Encefalocele } \\
\text { occipital }\end{array}$ & SNC & $\begin{array}{c}220 \\
0\end{array}$ & $\mathrm{HNN}$ \\
\hline 16 & 21 & $\begin{array}{l}\text { Nicaraguens } \\
\text { e, Alajuelita, } \\
\text { SJ }\end{array}$ & 37 & $x$ & & $x$ & $x$ & $\mathrm{x}$ & & $\begin{array}{c}\text { Sinusdermalis } \\
\text { Hipospadia }\end{array}$ & $\begin{array}{c}\text { SNC,G } \\
\mathrm{U}\end{array}$ & $\begin{array}{c}209 \\
5\end{array}$ & $\mathrm{HNN}$ \\
\hline 17 & 24 & $\begin{array}{c}\text { Nicaraguens } \\
\text { e-Santa } \\
\text { Ana, SJ }\end{array}$ & 39 & $x$ & & $x$ & $x$ & & $\mathrm{x}$ & $\begin{array}{l}\text { Atresia } \\
\text { tricuspídea+Ven } \\
\text { triculo Izq único }\end{array}$ & GU & $\begin{array}{c}254 \\
0\end{array}$ & $\mathrm{HNN}$ \\
\hline 18 & 26 & $\begin{array}{c}\text { Golfito,Punt } \\
\text { arenas }\end{array}$ & 36 & $\mathrm{x}$ & $x$ & & $x$ & & $\mathrm{x}$ & $\begin{array}{l}\text { Sd.Peters-Plus: } \\
\text { orejas implant } \\
\text { baja, } \\
\text { micrognatia,ma } \\
\text { mila separada }\end{array}$ & $\begin{array}{l}\text { Sínd. } \\
\text { Peters } \\
\text {-Plus }\end{array}$ & $\begin{array}{c}299 \\
0\end{array}$ & $\mathrm{HNN}$ \\
\hline 19 & 32 & $\begin{array}{l}\text { Santa Ana, } \\
\text { SJ }\end{array}$ & 36 & $\mathrm{x}$ & & $x$ & $x$ & & $\mathrm{x}$ & $\begin{array}{l}\text { Apéndices } \\
\text { preauric, } \\
\text { artorgriposis } \\
\text { MSIzq }\end{array}$ & $\begin{array}{l}\text { M. } \\
\text { Esquel } \\
\text { ético } \\
\text { Crane } \\
\text { o } \\
\text { facial }\end{array}$ & $\begin{array}{c}232 \\
5\end{array}$ & $\mathrm{HNN}$ \\
\hline 20 & 29 & Alajuelita, SJ & 40 & $x$ & & $x$ & $x$ & $x$ & & $\begin{array}{c}\text { Ano } \\
\text { imperforado }\end{array}$ & GI & $\begin{array}{c}367 \\
0\end{array}$ & $\mathrm{HNN}$ \\
\hline 21 & 28 & Central, SJ & 36 & $x$ & & $x$ & $x$ & & $\mathrm{x}$ & $\begin{array}{c}\text { Orejas implant } \\
\text { baja, frontal } \\
\text { amplio, mamilas } \\
\text { separadas, } 2^{\circ} \\
\text { dedo pie sobre } \\
\text { 1ero }\end{array}$ & $\begin{array}{l}\text { Sínd. } \\
\text { Down }\end{array}$ & $\begin{array}{c}249 \\
5\end{array}$ & HNN \\
\hline 22 & NC & $\begin{array}{l}\text { Nicaraguens } \\
\text { e, Escazú, SJ }\end{array}$ & 33 & $x$ & & $x$ & $x$ & & & $\begin{array}{l}\text { Labio y paladar } \\
\text { hendido }\end{array}$ & SNC & $\begin{array}{c}173 \\
5\end{array}$ & $\mathrm{HNN}$ \\
\hline 23 & 22 & Central, SJ & 38 & $x$ & & $x$ & $x$ & $x$ & & $\begin{array}{l}\text { Uropatía } \\
\text { articulada bil } \\
\text { compromete } \\
\text { hasta uréter }\end{array}$ & GU & $\begin{array}{c}294 \\
5\end{array}$ & HNN \\
\hline 24 & 25 & Mora, SJ & 39 & $x$ & & $x$ & $x$ & $x$ & & $\begin{array}{c}\text { Polidactilia pie } \\
\text { derecho }\end{array}$ & $\begin{array}{l}\text { Múscu } \\
\text { lo } \\
\text { Esquel } \\
\text { ético }\end{array}$ & $\begin{array}{c}308 \\
0\end{array}$ & Casa \\
\hline 25 & 20 & Mora, SJ & 39 & $x$ & & $x$ & $x$ & & $\mathrm{x}$ & $\begin{array}{l}\text { Secuencia } \\
\text { Potter, ano } \\
\text { imperforado }\end{array}$ & $\mathrm{GU}, \mathrm{GI}$ & $\begin{array}{c}159 \\
5\end{array}$ & $\begin{array}{l}\text { Falle } \\
\text { ce }\end{array}$ \\
\hline 26 & 20 & Puriscal, SJ & 36.4 & $\mathrm{x}$ & & $x$ & $x$ & & $\mathrm{x}$ & $\begin{array}{l}\text { Quiste cervical } \\
\text { D, Microtia D }\end{array}$ & $\begin{array}{c}\text { Crane } \\
0 \\
\text { facial }\end{array}$ & $\begin{array}{c}266 \\
5\end{array}$ & $\mathrm{HNN}$ \\
\hline
\end{tabular}




\begin{tabular}{|c|c|c|c|c|c|c|c|c|c|c|c|c|c|}
\hline 27 & 30 & $\begin{array}{c}\text { Nicaraguens } \\
\text { e, Central, } \\
\text { SJ }\end{array}$ & 37.5 & $x$ & - & - & $\mathrm{x}$ & & $x$ & $\begin{array}{l}\text { Anencefalia, } \\
\text { ano } \\
\text { imperforado, } \\
\text { criptorquidea } \\
\text { bilateral }\end{array}$ & $\begin{array}{l}\text { SNC, } \\
\text { GI,GU }\end{array}$ & $\begin{array}{c}299 \\
5\end{array}$ & $\begin{array}{l}\text { Falle } \\
\text { ce }\end{array}$ \\
\hline 28 & 33 & $\begin{array}{c}\text { Nicaraguens } \\
\text { eSanta Ana, } \\
\text { SJ }\end{array}$ & 40 & $x$ & & $x$ & $x$ & $x$ & & $\begin{array}{l}\text { Hoyuelo } \\
\text { preauric bil }\end{array}$ & $\begin{array}{l}\text { Crane } \\
\text { o } \\
\text { facial }\end{array}$ & $\begin{array}{c}299 \\
5\end{array}$ & Casa \\
\hline 29 & 41 & $\begin{array}{c}\text { Nicaraguens } \\
\text { e, Central, } \\
\text { SJ }\end{array}$ & 37 & $x$ & & $x$ & $x$ & & $x$ & $\begin{array}{c}\text { T-18, Atresia } \\
\text { Esófago }\end{array}$ & $\begin{array}{l}\text { Sind. } \\
\text { Edwar } \\
\text { d }\end{array}$ & $\begin{array}{c}182 \\
0\end{array}$ & $\begin{array}{l}\text { Falle } \\
\text { ce }\end{array}$ \\
\hline 30 & 14 & Central, SJ & 38 & $x$ & & $\mathrm{x}$ & $\mathrm{x}$ & $\mathrm{x}$ & & Polidactilia & $\begin{array}{l}\text { Múscu } \\
\text { lo } \\
\text { esquel } \\
\text { ético }\end{array}$ & $\begin{array}{c}364 \\
5\end{array}$ & Casa \\
\hline 31 & 27 & $\begin{array}{c}\text { Nicaraguens } \\
\text { eSanta } \\
\text { Ana,SJ }\end{array}$ & 38 & $x$ & & $\mathrm{x}$ & $\mathrm{x}$ & & $\mathrm{x}$ & $\begin{array}{l}\text { T-21, epicanto, } \\
\text { sandal gap, } \\
\text { occipucio plano, } \\
\text { hipotonía, }\end{array}$ & $\begin{array}{l}\text { Sínd. } \\
\text { Down }\end{array}$ & $\begin{array}{c}253 \\
5\end{array}$ & HNN \\
\hline 32 & 33 & $\begin{array}{c}\text { Nicaraguens } \\
\text { e,Mora, SJ }\end{array}$ & 39 & $x$ & & $\mathrm{x}$ & $\mathrm{x}$ & $x$ & & $\begin{array}{c}\text { Ventrículo } \\
\text { único, Mal } \\
\text { posicionamient } \\
\text { o de gdes vasos }\end{array}$ & CVC & $\begin{array}{c}329 \\
0\end{array}$ & $\mathrm{HNN}$ \\
\hline 33 & 34 & Central, SJ & 35.6 & $x$ & & $\mathrm{x}$ & $\mathrm{x}$ & & $x$ & $\begin{array}{l}\text { T-18, Frente } \\
\text { amplio, } \\
\text { hipertelorismo, } \\
\text { orejas bajas, } \\
\text { retrognatia, } \\
\text { mamilas } \\
\text { separadas,pies } \\
\text { mecedora, } \\
\text { criptorqui bil }\end{array}$ & $\begin{array}{l}\text { Sínd. } \\
\text { Edwar } \\
\text { d }\end{array}$ & $\begin{array}{c}167 \\
5\end{array}$ & $\begin{array}{l}\text { Falle } \\
\text { ce }\end{array}$ \\
\hline 34 & 20 & Alajuelita, SJ & 38.5 & $x$ & $x$ & & $x$ & & $x$ & T-21,CAV,CIV & $\begin{array}{l}\text { CVC } \\
\text { Sínd. } \\
\text { Down }\end{array}$ & $\begin{array}{c}385 \\
5\end{array}$ & \\
\hline 35 & 21 & $\begin{array}{c}\text { Desampara } \\
\text { dos, SJ }\end{array}$ & 40 & $x$ & & $x$ & $x$ & $\mathrm{X}$ & & $\begin{array}{l}\text { Encefalocele, } \\
\text { microcefalia }\end{array}$ & SNC & $\begin{array}{c}269 \\
0\end{array}$ & \\
\hline 36 & 38 & $\begin{array}{l}\text { Matina,Lim } \\
\text { ón }\end{array}$ & 41 & $x$ & $x$ & & $x$ & $x$ & & $\begin{array}{l}\text { Labio leporino y } \\
\text { paladar hendido }\end{array}$ & SNC & $\begin{array}{c}326 \\
5\end{array}$ & \\
\hline 37 & 27 & Central, SJ & 41 & $x$ & $x$ & & $x$ & $\mathrm{X}$ & & $\begin{array}{c}\text { Hernia } \\
\text { diafragmatica }\end{array}$ & GI & $\begin{array}{c}348 \\
3\end{array}$ & \\
\hline 38 & 25 & $\begin{array}{c}\text { Pérez } \\
\text { Celedón, SJ }\end{array}$ & 29 & $x$ & $x$ & & $x$ & $X$ & & $\begin{array}{l}\text { Frente amplia, } \\
\text { orejasbajas, } \\
\text { mamilas } \\
\text { separadas, pie }\end{array}$ & $\begin{array}{l}\text { Sind. } \\
\text { Down }\end{array}$ & $\begin{array}{c}119 \\
0\end{array}$ & $\begin{array}{l}\text { Falle } \\
\text { ce }\end{array}$ \\
\hline
\end{tabular}




\begin{tabular}{|c|c|c|c|c|c|c|c|c|c|c|c|c|c|}
\hline & & & & & & & & & & $\begin{array}{l}\text { mecedora,super } \\
\text { posic 5to artejo } \\
\text { en 4to }\end{array}$ & & & \\
\hline 39 & 34 & $\begin{array}{c}\text { Pérez } \\
\text { Celedón, SJ }\end{array}$ & 35 & $x$ & & $x$ & $x$ & $\mathrm{X}$ & & $\begin{array}{c}\text { T-18 x } \\
\text { cariotipo,usgper } \\
\text { inatal displasia } \\
\text { renal }\end{array}$ & $\begin{array}{l}\text { Sind. } \\
\text { Edwar } \\
\quad \text { d }\end{array}$ & $\begin{array}{c}233 \\
0\end{array}$ & \\
\hline 40 & 26 & Mora, SJ & 40 & $x$ & & $x$ & $x$ & $\mathrm{x}$ & & Raquisquisis & SNC & $\begin{array}{c}311 \\
5\end{array}$ & HNN \\
\hline 41 & 41 & $\begin{array}{l}\text { Nicaraguens } \\
\text { e, Santa } \\
\text { Ana, SJ }\end{array}$ & 36 & $x$ & - & - & $x$ & & $x$ & $\begin{array}{c}\text { Escoliosis, } \\
\text { dedos en tijera, } \\
\text { luxación } \\
\text { bilateral, ano } \\
\text { imperforado, } \\
\text { genitales } \\
\text { ambiguos, } 2 \\
\text { vasos umbilic }\end{array}$ & $\begin{array}{l}\text { Múscu } \\
\text { lo } \\
\text { esquel } \\
\text { ética } \\
\text { GI } \\
\text { GU } \\
\text { CVC }\end{array}$ & $\begin{array}{c}216 \\
0\end{array}$ & $\begin{array}{l}\text { Falle } \\
\text { ce }\end{array}$ \\
\hline 42 & 18 & Escazú, SJ & 40 & $x$ & $x$ & & $x$ & $x$ & & Espina bífida & SNC & $\begin{array}{c}374 \\
5\end{array}$ & HNN \\
\hline 43 & 25 & Mora, SJ & 40.6 & $x$ & $\mathrm{x}$ & & $x$ & $\mathrm{x}$ & & Sinus dermalis & SNC & $\begin{array}{c}321 \\
0\end{array}$ & Casa \\
\hline 44 & 26 & Alajuelita,SJ & 34 & $x$ & & $x$ & $x$ & $\mathrm{x}$ & & Hipospadia & GU & $\begin{array}{c}205 \\
5\end{array}$ & \\
\hline 45 & 18 & Central, SJ & 36 & $\mathrm{x}$ & $\mathrm{x}$ & & $x$ & $x$ & & $\begin{array}{c}\text { Fístula preauric } \\
\text { izq }\end{array}$ & $\begin{array}{l}\text { Crane } \\
\text { o } \\
\text { facial }\end{array}$ & $\begin{array}{c}208 \\
5\end{array}$ & Casa \\
\hline 46 & 20 & Escazú, SJ & 38 & $\mathrm{x}$ & & $x$ & $x$ & & $\mathrm{x}$ & $\begin{array}{l}\text { Mielomeningoc } \\
\text { ele, orejas } \\
\text { bajas, } \\
\text { retrognatia, } \\
\text { cuello corto, } \\
\text { hidrocefalia, } \\
\text { frente } \\
\text { prominente }\end{array}$ & $\begin{array}{l}\text { SNC } \\
\text { Síndro } \\
\text { mes }\end{array}$ & $\begin{array}{c}264 \\
0\end{array}$ & HNN \\
\hline 47 & 37 & $\begin{array}{c}\text { Curridabat, } \\
\text { SJ }\end{array}$ & 34 & $x$ & $\mathrm{x}$ & & $x$ & $x$ & & Fenotipo Down & $\begin{array}{l}\text { Sind. } \\
\text { Down }\end{array}$ & $\begin{array}{c}162 \\
0\end{array}$ & \\
\hline 48 & NC & Escazú, SJ & 38 & $\mathrm{x}$ & & $x$ & $x$ & $x$ & & $\begin{array}{l}\text { Apéndice lat } 5 \text { to } \\
\text { ortejo }\end{array}$ & Otras & $\begin{array}{c}282 \\
5\end{array}$ & \\
\hline 49 & 21 & Alajuelita, SJ & 37.1 & $x$ & $x$ & & $x$ & $x$ & & $\begin{array}{c}\text { Bandas } \\
\text { amniótica } \\
\text { amputación } \\
\text { metacarp } \\
\text { 2|dedomano } \\
\text { izq, elefantiasis }\end{array}$ & $\begin{array}{l}\text { Múscu } \\
\text { lo } \\
\text { esquel } \\
\text { ética }\end{array}$ & $\begin{array}{c}276 \\
0\end{array}$ & \\
\hline
\end{tabular}




\begin{tabular}{|c|c|c|c|c|c|c|c|c|c|c|c|c|c|}
\hline & & & & & & & & & & $\begin{array}{l}\text { falange distal } \\
\text { del 1er dedo } D\end{array}$ & & & \\
\hline 50 & 33 & Central, SJ & 40 & $\mathrm{x}$ & & $x$ & $x$ & $\mathrm{x}$ & & $\begin{array}{l}\text { Apéndice } \\
\text { preauric }\end{array}$ & $\begin{array}{c}\text { Crane } \\
0 \\
\text { facial }\end{array}$ & $\begin{array}{c}389 \\
0\end{array}$ & Casa \\
\hline 51 & 17 & Central, SJ & 40 & $\mathrm{x}$ & & $x$ & $x$ & & $\mathrm{x}$ & $\begin{array}{l}\text { Hipospadia, } \\
\text { Criptorquidea }\end{array}$ & GU & $\begin{array}{c}266 \\
5\end{array}$ & Casa \\
\hline 52 & 30 & Central, SJ & 35 & $\mathrm{x}$ & & $x$ & $x$ & & $\mathrm{x}$ & $\begin{array}{c}\text { Displasia cadera } \\
\text { D, } \\
\text { Sindectilia } 2^{\circ}, 3^{\circ} \\
\text { pie D }\end{array}$ & $\begin{array}{l}\text { Múscu } \\
\text { lo } \\
\text { esquel } \\
\text { ética }\end{array}$ & $\begin{array}{c}247 \\
5\end{array}$ & Casa \\
\hline 53 & 21 & Escazú, SJ & 26 & $x$ & & $x$ & $x$ & $x$ & & $\begin{array}{l}\text { Atresia } \\
\text { esofágica }\end{array}$ & GI & 620 & $\begin{array}{l}\text { Falle } \\
\text { ce }\end{array}$ \\
\hline 54 & 28 & NC & 27 & $\mathrm{x}$ & $\mathrm{x}$ & & $x$ & & $\mathrm{X}$ & Dx no específico & & 925 & $\begin{array}{l}\text { Falle } \\
\text { ce }\end{array}$ \\
\hline
\end{tabular}

Fuente: Neonatología, HSJD.

Tabla II. Rango de edad de las madres de recién nacidos con malformaciones congénitas en HSJD de Enero a Diciembre 2014, según Neonatología HSJD

\begin{tabular}{|c|c|c|}
\hline \multicolumn{2}{|c|}{$\begin{array}{l}\text { Tabla II } \\
\text { Rango de edad de las madres de recién nacidos con malformaciones congénitas en HSJD de Enero a } \\
\text { Diciembre 2014, según Neonatología HSJD }\end{array}$} \\
\hline RANGO DE EDAD (años) & CASOS & \% \\
\hline $\mathbf{1 5}$ & 2 & 19.2 \\
\hline $\mathbf{1 6 - 2 0}$ & 10 & 26.9 \\
\hline $\mathbf{2 1 - 2 5}$ & 14 & 25 \\
\hline $\mathbf{2 6 - 3 0}$ & 13 & 17.3 \\
\hline $\mathbf{3 1 - 3 5}$ & 9 & 7.6 \\
\hline
\end{tabular}

Fuente: Neonatología, HSJD. 
Tabla III. Distribución geográfica de las anomalías congénitas atendidas en el HSJD de Enero a Diciembre 2014, según Neonatología HSJD

\begin{tabular}{|c|c|c|}
\hline \multicolumn{3}{|c|}{$\begin{array}{l}\text { Distribución geográfica de las anomalías congénitas atendidas en el HSJD de Enero a } \\
\text { Diciembre 2014, según Neonatología HSJD }\end{array}$} \\
\hline CANTÓN & CASOS & $\%$ \\
\hline Central, SanJosé & 14 & 26.4 \\
\hline Santa Ana, San José & 10 & 18.8 \\
\hline Alajuelita, San José & 7 & 13.2 \\
\hline Escazú, San José & 7 & 13.2 \\
\hline Mora, San José & 5 & 9.4 \\
\hline Puriscal, San José & 2 & 3.7 \\
\hline Pérez Zeledón, San José & 2 & 3.7 \\
\hline Curridabat, San José & 1 & 1.8 \\
\hline Desamparados, San José & 1 & 1.8 \\
\hline Golfito,Puntarenas & 1 & 1.8 \\
\hline Buenos Aire, Puntarenas & 1 & 1.8 \\
\hline Osa, Puntarenas & 1 & 1.8 \\
\hline Unión, Cartago & 1 & 1.8 \\
\hline
\end{tabular}

Fuente: Neonatología, HSJD

Tabla IV. Edad gestacional de interrupción del embarazo en productos con mal-formaciones congénitas en HSJD de Enero a Diciembre 2014, según Neonatología HSJD

\section{Tabla IV}

Edad gestacional de interrupción del embarazo en productos con malformaciones congénitas en HSJD de Enero a Diciembre 2014, según Neonatología HSJD

\begin{tabular}{|c|c|c|}
\hline$<34$ SEMANAS & 5 & $9.2 \%$ \\
\hline 34.1-36.6 SEMANAS & 14 & $25.9 \%$ \\
\hline 37-41 SEMANAS & 35 & $64.8 \%$ \\
\hline
\end{tabular}


Tabla V. Incidencia de malformaciones congénitas por sistema en el HSJD durante Enero a Diciembre 2014, según Neonatología HSJD

\begin{tabular}{|c|c|c|}
\hline \multicolumn{2}{|c|}{$\begin{array}{c}\text { Tabla V } \\
\text { Incidencia de malformaciones congénitas por sistema en el HSJD durante Enero a Diciembre 2014, } \\
\text { según Neonatología HSJD }\end{array}$} \\
\hline MALFORMACIÓN & CASOS & PORCENTAJE \% \\
\hline SISTEMA NERVIOSO CENTRAL & 14 & 22.9 \\
\hline SISTEMA CARDIOVASCULAR & 5 & 8.1 \\
\hline SISTEMA GASTROINTESTINAL & 9 & 14.7 \\
\hline SISTEMA GENITO URINARIO & 10 & 16.3 \\
\hline SISTEMA MÚSCULO & 10 & 16.3 \\
ESQUELÉTICO & 1 & 1.6 \\
\hline SISTEMA RESPIRATORIO & 3 & 4.9 \\
\hline CRANEOFACIALES & 9 & 14.7 \\
\hline SÍNDROMES & & \\
\hline
\end{tabular}

Fuente: Neonatología, HSJD

Tabla VI. Incidencia de malformaciones congénitas por sistema en el HSJD durante Enero a Diciembre 2014, según Neonatología HSJD

\section{Tabla VI}

Sexo del recién nacido con malformaciones congénitas durante Enero a Diciembre 2014 en HSJD, según Neonatología HSJD

\begin{tabular}{|c|c|c|}
\hline MASCULINO & 33 & 61.1 \\
\hline FEMENINO & 19 & 35.2 \\
\hline AMBIGUO & 2 & 3.7 \\
\hline
\end{tabular}

Fuente: Neonatología, HSJD 